\title{
Significance of Combined Imaging BI-RADS Assessment in Breast Cancer and Pathology Comparison
}

\author{
Min LIU ${ }^{1}$, Zhi-Hou ZHOU ${ }^{1}$, Ying $\mathrm{LI}^{2}$, Jing-Zhong $\mathrm{XU}^{3}$, \\ Gang-Ping WANG ${ }^{4,}$, , \\ ${ }^{1}$ Department of Radiology, Rizhao People's Hospital, Rizhao, P.R.China \\ ${ }^{2}$ Department of Pharmacy, Rizhao People's Hospital Rizhao, P.R.China \\ ${ }^{3}$ Department of Traditional Chinese Medicine, Jufeng Hospital, Rizhao,P.R.China \\ ${ }^{4}$ Department of Pathology, Rizhao People's Hospital, Rizhao, P.R.China \\ aemail: wgprzph93@126.com \\ ${ }^{*}$ Corresponding author
}

Keywords: Breast cancer, BI-RADS, Mammography, Ultrasonography, Diagnosis, Differential diagnosis.

\begin{abstract}
Mammography (MG) is the only proven modality to reduce mortality of breast cancer and has been shown to reduce mortality from breast cancer, and ultrasonography (US) is a well-known adjunct to screening MG. BI-RADS classification is actually practical and correlation with histopathology on the palpable diagnostic cases. To explore the clinical value of combined detection of MG and US BI-RADS in the diagnosis of breast cancer, we examined 212 patients using MG and US (62 cases breast cancer and 150 cases benign leision). All selected cases were re-evaluated by BI-RADS MG alone, US alone, and combined MG and US. In the combined imaging assessment, BI-RADS 1-4a category was MG and US both no more than $4 \mathrm{a}$, however, $4 \mathrm{~b}-5 \mathrm{a}$ cacategory was considered as the score of MG and US both more than 4a. MG and US alone findings BI-RADS 1-4a level were 142 cases and 144 cases, respectively; 4b-5 level were 70 and 68, respectively; and for Combination, there were 166 cases $1-4 \mathrm{a}$ and 46 cases $4 \mathrm{~b}-5$ level. Our results demonstrated that the sensitivity of MG, US alone was $80.65 \%$ and $72.58 \%$, respectly; and specificity was $86.67 \%$ and $84.67 \%$, respectly. The sensitivity of MG and US in combination was $69.35 \%$, however, the specificity was improved into $98.00 \%$, and a positive predictive value was improved into $93.47 \%$. The differences in specificity and positive predictive values between MG and combined imaging assessment, US and combined all were statistically significant $(P<0.05)$. The specificity and positive predictive values of combined assessment was the highest, and the sensitivity and negative predictive values was the highest in the MG assessment. Combined imaging assessment is more effective in diagnosing breast lesions.
\end{abstract}

\section{Introduction}

Breast carcinoma is a serious threat to women's health [1]. In China, the incidence of breast cancer is relatively high, and the peak incidence is in advance [2,3]. Mammography (MG) has been shown to reduce mortality from breast cancer, and ultrasonography (US) is a well-known adjunct to screening MG [4-5]. Mammographic screening is the only proven modality to reduce mortality of breast cancer, with the rates ranging from 10 to $30 \%$ [6-8]. The American College of Radiology (ACR) breast 
imaging reporting and data system blood (BI-RADS) classification is actually practical and correlation with histopathology and combined uses with triple assessment (examination, imaging, and biopsy) on the palpable diagnostic cases. Five levels are included in BI-RADS, and level 4 is divided into three sublevels of $4 \mathrm{a}, 4 \mathrm{~b}$ and $4 \mathrm{c}$. Lesion less than $4 \mathrm{a}$ is considered as a benign lesion while lesion more than $4 \mathrm{a}$ is considered as malignant lesions. The ACR BI-RADS provides standardized descriptors of imaging features of breast lesions, it is also helpful in predicting benign or malignant potential, and can be used globally. To validate indicate different malignancy incidence rates and whether combined detaction may improve accuracy and sensitivity of predicting diagnosis of breast cancer, in this study, we composed three different assessments of MG alone, US alone and combined score diagnostic value was investigated.

\section{Patients and Methods}

\section{Patients}

The present study was approved by the ethical committee at Rizhao People's Hospital. Written informed consent was obtained from all of the patients before their participation in the current study. The study was a retrospective study, and was conducted on 212 women aged 40-70 years of age in the period from January 2011 to December 2014at Rizhao people's Hospital, China, and a complete clinical and follow-up data were confirmed by surgery and pathology. We excluded cases in which both MG and US were not performed. Preoperatively, the BIRADS breast lesions detected by routine MG and US were used. Postoperatively, the breast lesions were diagnosed as benign and malignant lesions according to pathological results. Finally, totally 212 cases were finally selected for this study. Patients were divided into two groups in radiological findings ACR BI RADS 1-4a and $4 b-5$ as the study and the control group. If the patients underwent more than one imaging examination before tissue biopsy, the latest one was analyzed. In patients with bilateral biopsies or more than one biopsy in one breast, the most serious result was considered. All selected cases were re-evaluated by our 5-point score with the following assessments: MG alone, US alone, and combined MG and US. In the combined imaging assessment, BI-RADS 1-4a category was MG and US alone both no more than 4a, however, 4b-5a cacategory was considered as the score of MG and US both more than 4a. For the MG alone, US alone, and combined imaging scores, scores $1-4 \mathrm{a}$ indicated negative for cancer and scores $4 \mathrm{~b}-5$ regarded as positive for cancer. The patients consisted of 62 patients in whom breast cancer had been histologically verified and 150 patients benign lesion.

\section{Imaging Protocols}

MG and US was interpreted by experienced technologists, and the findings were reported by 2 experienced radiologists under ACR BI-RADS categories. Diagnostic mammograms were obtained using standard craniocaudal (CC) and mediolateral oblique (MLO) views by well-trained technologists using digital MG machines with full-field digital mammograms, Senographe 2000 D, GE, USA. Standard mammographic projections were made: craniocaudal and mediolateral. All US examinations included real-time bilateral whole-breast and power Doppler blood flow scans, using US machines, iu Elite Medical System, Philips, USA, , with linear probes measuring 5-12 MHz. Mammography and breast ultrasound findings were classified by 
BI RADS classification into one of five categories: 1. Breasts where no pathological lesions are seen; 2. Benign findings; 3 . Probably benign findings; 4. Lesions suspicious for malignancy, And, level 4 is divided into three sublevels of $4 \mathrm{a}, 4 \mathrm{~b}$ and $4 \mathrm{c}$.; 5 . Lesions highly suspicious for malignancy-malignant lesion. And, level 4 is divided into three sublevels of $4 \mathrm{a}, 4 \mathrm{~b}$ and $4 \mathrm{c}$. Lesion less than $4 \mathrm{a}$ is considered as a benign lesion while lesion more than $4 \mathrm{a}$ is considered as malignant lesions.

\section{Pathology Study}

Tissue samples were fixed in $10 \%$ neutral buffered formalin and embedded in paraffin. Tissue sections were deparaffinized and rehydrated using standard procedures. The pathological categorization was determined according to the current World Health Organization classification system (WHO 2012) [1] and the pathological diagnosis was verified by histological methods independently by two pathologists. The pathological reading was determined for each slide with an overall pathological diagnosis determined for each subject. Assessment of the staining was evaluated by two independent pathologists without knowledge of the clinical status of the patients.

\section{Statistical Analysis}

SPSS version 17.0 statistical software (SPSS Inc.: Chicago, IL, USA) was used to analyze the data. Enumeration data with $\chi^{2}$ test. The difference between the samples was considered significant when $P$ was less than 0.05 .

\section{Results}

Two hundrend and twelve patients were enrolled in this study, aged 40-70 years of age with an average age of $52.16 \pm 7.43$ years. The BI-RADS breast lesions detected by routine MG and US were used. The diagnostic indicators with high sensitivity and specificity were tumor edge, enhanced range and score of elastography (Fig. 1). Factors of tumor edge, enhanced order, contrast mode and score of elastography were related with the benign and malignant features of breast lesions. In the group of patients there were 62 cases breast cancer and 150 cases benign leision

Statistical analysis of the differences in the distributions of breast lesions detected by the different examination methods MG alone, US alone and combined MG and US in BI-RADS classifying diagnosis was performed. The results were indicated in Table 1. Preoperative MG or US alon findings BI-RADS 1-4a level were 142 cases and 144 cases, respectively; BI-RADS 4b-5 level were 70 and 68, respectively; and for combined MG and US imaging scores, there were 166 cases 1-4a and 46 cases BI-RADS 4b-5 level.

The diagnostic discriminative value in breast cancer was shown in Table 2. Our results demonstrated that the sensitivity of MG, US alone was $80.65 \%$ and $72.58 \%$, respectly; and specificity was $86.67 \%$ and $84.67 \%$, respectly, and sensitivity of MG and US in combination was $69.35 \%$, however, the specificity was improved into $98.00 \%$, positive predictive values (PPV) was improved into $93.47 \%$. The specificity and positive predictive values of combined imaging assessment was the highest, and the sensitivity and negative predictive values (NPV) was the highest in the MG assessment. The differences in specificity and positive predictive values between MG and combined imaging assessment, US and combined all were statistically significant $(P<0.05)$. Combined imaging assessment is more effective in diagnosing breast lesions. 

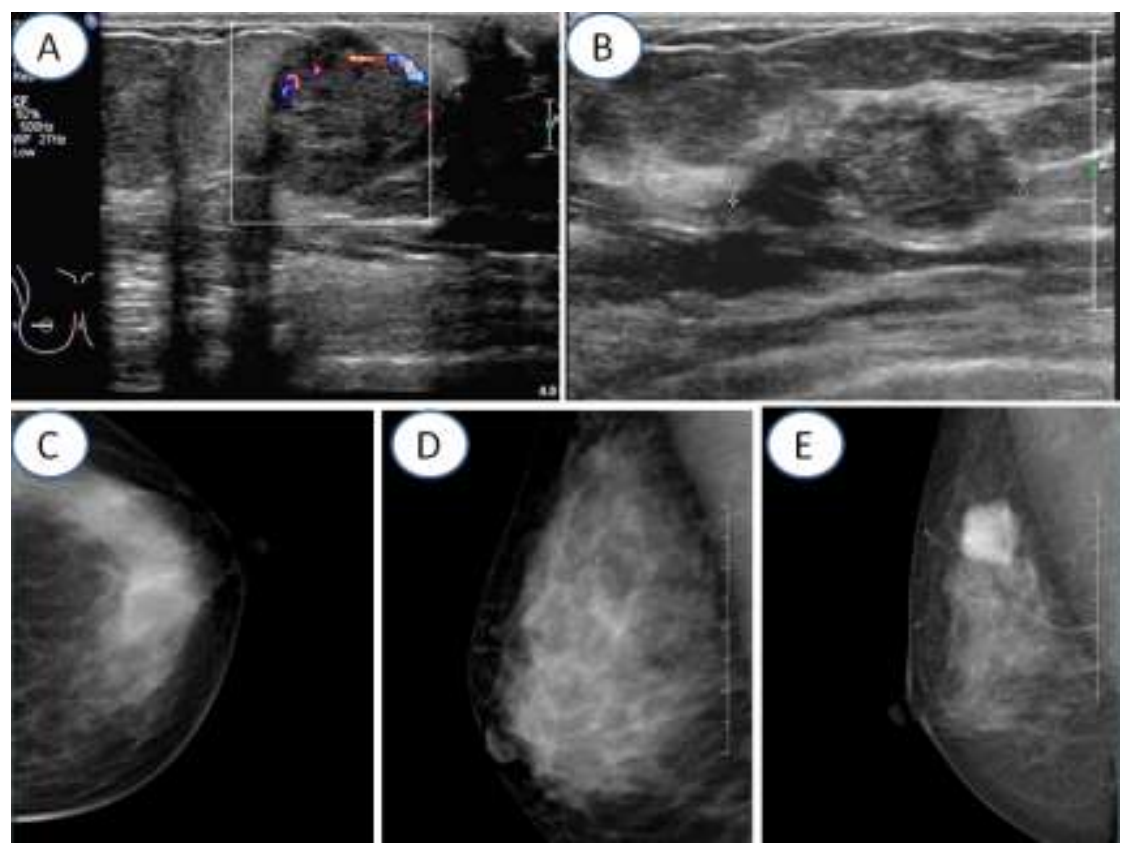

Figure 1. The BI-RADS breast lesions detected by routine MG and US. A US-detected invasive ductal carcinoma assessed as BI-RADS category 4A; B, US-detected BI-RADS category 5 (invasive ductal carcinoma); C MG - detected 3 (benign lesion); D, MG -detected 4A (invasive ductal carcinoma); E, MG -detected 4C (invasive ductal carcinoma)

Table 1. Case of malignancy incidence for each group of categories.

\begin{tabular}{ccccc}
\hline BI-RADS assessment & & No of patient & Benign & Malignancy \\
\hline MG alone & $1-4 \mathrm{a}$ & 212 & 150 & 62 \\
& $4 \mathrm{~b}-5$ & 142 & 130 & 50 \\
US alone & & 70 & 20 & 12 \\
& $1-4 \mathrm{a}$ & 144 & 127 & 45 \\
Combination* & $4 \mathrm{~b}-5$ & 68 & 23 & 19 \\
& & & & 43 \\
\hline
\end{tabular}

*MG, mammography; US, ultrasonography; Combined MG \& US, the category was determined by BI-RADS 1-4a category was MG and US alone both no more than 4a, 4b-5a cacategory was considered as the score of MG and US both more than $4 \mathrm{a}$.

Table 2. Comparison of Results by Different Assessment Methods.

\begin{tabular}{lllll}
\hline \multicolumn{1}{c}{ Groups } & Sensitivity (\%) & Specificity (\%) & PPV (\%) & NPV (\%) \\
\hline MG alone & 80.65 & 86.67 & 71.43 & 91.55 \\
US alone & 72.58 & 84.67 & 66.18 & 84.67 \\
Combination & 69.35 & $98.00^{*}$ & $93.47 *$ & 88.55 \\
\hline
\end{tabular}

*Compared with other items, $\mathrm{P}<0.01$.

MG, mammography; US, ultrasonography; Combined MG \& US, the category was determined by BI-RADS 1-4a category was MG and US alone both no more than 4a, however, 4b-5a cacategory was considered as the score of MG and US both more than 4a; PPV, positive predictive values; NPV, negative predictive values. 


\section{Discussion}

In China, the incidence of breast cancer is relatively high, and the peak incidence is in advance, young breast cancer has become a major clinical type of breast cancer, a large number of patients died of breast cancer complications or serious organ metastasis each year [2-3,9]. The incidence rate of breast cancer in China was 2.55/100,000 in 2009, accounting for $16.81 \%$ of female malignancies, while the five year disease-free survival in patients with breast cancer has increased from $70 \%$ in 1980 to $85 \%$ in 2011 [3,10]. In 2003, the breast imaging report and data system (BI-RADS) was issued by the American College of Radiology to standardize mammographic reporting [11]. Five levels are included in BI-RADS. And, level 4 is divided into three sublevels of $4 \mathrm{a}, 4 \mathrm{~b}$ and $4 \mathrm{c}$. Lesion less than $4 \mathrm{a}$ is considered as a benign lesion while lesion more than $4 \mathrm{a}$ is considered as malignant lesions. The routine sonographic manifestations of level 4 BI-RAD breast lesions tend to have a certain degree of overlapping and are sometimes difficult to judge [12-14]. Thus, it is difficult to identify the nature of such lesions in clinic. Screening US, however, has been devaluated due to its operator dependency, dubious cost-effective (ness, and relatively high false positive rate [13-16]. In this study, combined MG and US, the category was determined by BI-RADS 1-4a category was MG and US alone both no more than $4 \mathrm{a}$, however, $4 \mathrm{~b}-5 \mathrm{a}$ cacategory was considered as the score of MG and US both more than 4a; PPV, positive predictive values; NPV, negative predictive values. ACR BI-RADS classification is actually practical and correlation with histopathology and combined uses with triple assessment-examination, imaging, and biopsy-on the palpable diagnostic cases. Our study shows that the specificity and positive predictive values of combined imaging assessment was the highest, and the sensitivity and negative predictive values (NPV) was the highest in the MG assessment. The differences in specificity and positive predictive values between MG and combined imaging assessment, US and combined all were statistically significant $(P<0.05)$. Combined imaging assessment is more effective in diagnosing breast lesions. The cancer incidence might be different whether it is first-round screening or subsequent screening and according to the risk of different breast cancers.

Although combined imaging assessment has a definite detection benefit, this diagnostic method is applicable for screening purposes to women with unelevated risk of breast cancer, there are several limitations in our study. First, the cost of combined imaging assessment is not so attractive to patients. In addition, mammography is not to be effective in dense breasts and not perfect for young women. Third, combined imaging assessment there would be some ionizing radiation in adition mammography screening, and there is some patient discomfort.

\section{Conflict of Interest}

The authors declare no conflicts of interest.

\section{Acknowledgements}

The authors acknowledge financial support from the Application Technology Research and Development Project Foundation in Rizhao City (No. 2014SZSH02), Medicine and Health Care Science and Technology Development Plan Projects Foundation of Shandong Province (No. 2014WS0282, 2014WSA11003), as well as the Medical Staff' Science and Technology Innovation Projects of Shandong Province Medical Union Committee (No. 201515). 


\section{References}

[1] Lakhani SR, Ellis IO, Schnitt SJ, et al. WHO classification of tumours of the breast [M]. Lyon, France: IARC Press, 2012: pp1-117.

[2] Wang G, Qin M, Liang Y. The significance of biomarkers in nipple discharge and serum in diagnosis of breast cancer. In: Liquan Xie, Dianjian Huang, eds. Advanced Engineering and Technology; CRC Press2014: pp665-671.

[3] Chen W, Zheng R, Zeng H, Zhang S, He J. Annual report on status of cancer in China, 2011. Chin J Cancer Res 2015; 27: 2-12.

[4] Kelly KM, Dean J, Lee SJ, Comulada WS. Breast cancer detection: radiologists' performance using mammography with and without automated whole-breast ultrasound. Eur Radiol 2010; 20 (11):2557-64. doi: 10.1007/s00330-010-1844-1 PMID: 20632009; PubMed Central PMCID: PMC2948156.

[5] Chan SW, Cheung PS, Chan S, Lau SS, Wong TT, Ma M, et al. Benefit of ultrasonography in the detection of clinically and mammographically occult breast cancer. World J Surg 2008; 32 (12):2593-8. doi: 10.1007/s00268-007-9273-2 PMID: 17960454.

[6] Kalager M, Zelen M, Langmark F, Adami HO. Effect of screening mammography on breast-cancer mortality in Norway. N Engl J Med 2010;363:1203-10.

[7] Lee CH, Dershaw DD, Kopans D, Evans P, Monsees B, Monticciolo D, et al. Breast cancer screening with imaging: recommendations from the Society of Breast Imaging and the ACR on the use of mammography, breast MRI, breast ultrasound, and other technologies for the detection of clinically occult breast cancer. J Am Coll Radiol 2010;7:18-27.

[8] Smart CR, Byrne C, Smith RA, Garfinkel L, Letton AH, Dodd GD, et al. Twenty-year follow-up of the breast cancers diagnosed during the Breast Cancer Detection Demonstration Project. CA Cancer J Clin 1997;47:134-49.

[9] Wang G, Zhang H, Zhang Z. Clinical and pathological characteristics of intraductal proliferative lesions and coexist with invasive ductal carcinomas.Chinses-German J Clin Oncol 2013; 12: 574-80.

[10] Wang G, Qin Y, Zhang J, Zhao J, Liang Y, Zhang Z, et al. Nipple Discharge of CA153, CA125, CEA and TSGF as a New Biomarker Panel for Breast Cancer. Int. J. Mol. Sci.2014;15:9546-65.

[11] Sippo DA, Warden GI, Andriole KP, Lacson R, Ikuta I, Birdwell RL, Khorasani R. Automated extraction of BI-RADS final assessment categories from radiology reports with natural language processing. J Digit Imaging 2013; 26: 989-94.

[12] Raza S, Chikarmane SA, Neilsen SS, Zorn LM, Birdwell RL. BI-RADS 3, 4, and 5 lesions: value of US in management follow-up and outcome. Radiology 2008; 248 : 773-81.

[13] Leng X, Huang G, Yao L, Ma F. Role of multi-mode ultrasound in the diagnosis of level 4 BI-RADS breast lesions and Logistic regression mode Int J Clin Exp Med 2015;8:15889-99. 
[14] Heinig J, Witteler R, Schmitz R, Kiesel L, Steinhard J. Accuracy of classification of breast ultrasound findings based on criteria used for BI-RADS. UItrasound Obstet Gynecol 2008; 32: 573-8.

[15] Lazarus E, Mainiero MB, Schepps B, Koelliker SL, Livingston LS. BI-RADS lexicon for US and mammography: interobserver variability and positive predictive value. Radiology 2006;239:385-91.

[16] Magee BD. Ultrasound and mammography for breast cancer screening. JAMA 2008;300:1514. 